\title{
BIODEGRADACIÓN AEROBIA DE FRACCIONES DE HIDROCARBUROS PROVENIENTES DE LA ACTIVIDAD PETROLERA EN UN SUELO DE LA REGIÓN PATAGONIA NORTE, ARGENTINA
}

\author{
Cecilia E. Silvana ALVARO ${ }^{1 *}$, Lucas Agustín AROCENA², Miguel Ángel MARTÍNEZ ${ }^{3}$ y \\ Norma Ethel S. NUDELMAN ${ }^{4}$
}

${ }^{1}$ Laboratorio de Recuperación de Suelos Regionales, Facultad de Ingeniería-Instituto de Investigaciones y Desarrollo en Ingeniería de Procesos, Biotecnología y Energías Alternativas, Universidad Nacional del Comahue. Buenos Aires 1400, Neuquén (8300), Argentina

${ }^{2}$ Laboratorio de Recuperación de Suelos Regionales, Facultad de Ingeniería, Universidad Nacional del Comahue. Buenos Aires 1400, Neuquén (8300), Argentina

${ }^{3}$ Facultad de Tecnología y Ciencia de los Alimentos, Universidad Nacional del Comahue. 25 de mayo y Reconquista, Villa Regina, Río Negro (8336), Argentina,

${ }^{4}$ Facultad de Ciencias Exactas y Naturales, Universidad de Buenos Aires. Pabellón II, Piso 3, Ciudad Universitaria, Buenos Aires (1428), Argentina

*Autor para correspondencia: silvana_alvaro@hotmail.com

(Recibido abril 2016; aceptado agosto 2016)

Palabras clave: biorremediación, petróleo, explotación, biosólidos, suelos norpatagónicos, microorganismos nativos

\section{RESUMEN}

Las técnicas recientes de evaluación de sitios contaminados con hidrocarburos se fundamentan en la metodología del análisis de riesgo. El efecto sobre la salud y el ecosistema que producen agentes químicos presentes en un ambiente contaminado es estimado como la sumatoria de riesgos individuales, siendo la concentración de hidrocarburos un dato relevante. La biorremediación es una tecnología sustentable que acelera la degradación del petróleo en suelos contaminados y posee una relación costo beneficio favorable frente a otros métodos de remediación. El presente trabajo, realizado a escala de laboratorio, estudia la velocidad de degradación de distintas fracciones del petróleo mediante la incorporación de biosólidos (lodos cloacales) a un suelo contaminado de la Norpatagonia Argentina. Se determinó la concentración de hidrocarburos totales y el recuento de microorganismos presentes en el suelo al iniciar y finalizar el proceso de biorremediación. La degradación de las fracciones de hidrocarburos se analizó a intervalos regulares del proceso. Los resultados obtenidos mostraron una marcada disminución en la concentración de alcanos e hidrocarburos aromáticos de menor peso molecular a los 14 días de tratamiento. Los hidrocarburos de fracción pesada, resistentes a la biodegradación, mostraron reducciones significativas durante todo el proceso; su baja volatilidad y escasa migración por lixiviación indicarían que la degradación se debió principalmente al metabolismo de los microorganismos nativos presentes en suelos norpatagónicos. La comparación con el tratamiento convencional indica que la adición de biosólidos mejoró la remediación y que la biodiversidad de microorganismos presentes en el suelo es un factor importante para lograr una degradación significativa de fracciones del petróleo. 
Key words: bioremediation, petroleum exploitation, biosolids, north Patagonian soils, native microorganisms

\begin{abstract}
Recent techniques for assessing hydrocarbon contaminated sites are mainly based on risk analysis methodology. The effects of chemicals present in contaminated sites on the human health and the ecosystem are usually estimated as the addition of individual risks. In this sense, the hydrocarbon concentration is a relevant data. Bioremediation is a sustainable technology that accelerates the full or partial degradation of petroleum in contaminated soils and it shows a suitable cost-benefit ratio over other remediation methods. This work describes a laboratory scale study of the degradation rate for petroleum fractions by the incorporation of "biosolids" (sewage sludge) in an oil contaminated Patagonian soil. The determination of the total hydrocarbon concentration and the count of microorganisms in soil were performed both at the beginning and the end of the bioremediation process. The hydrocarbon degradation was analyzed at regular intervals during the study. The observed results showed that alkanes and lower molecular weight aromatic hydrocarbon concentrations were negligible after 14 days of treatment. The heavy hydrocarbon fraction, considered recalcitrant to biodegradation, was also significantly reduced during the biodegradation process. Since heavy hydrocarbons have low volatility and scarse migration by leaching, the observed decrease indicates that heavy hydrocarbon elimination could be mainly due to the native microorganism activity in the north Patagonian soils. Comparison with conventional treatment indicates that addition of "biosolids" enhances hydrocarbon remediation and that the biodiversity of microorganisms in soils is an important factor in achieving significant petroleum degradation.
\end{abstract}

\section{INTRODUCCIÓN}

La actividad hidrocarburífera en la Provincia del Neuquén, Argentina, constituye la labor productiva más importante asociada al cobro de regalías, generación de empleo y actividades comerciales subsecuentes a las etapas de exploración y explotación que determinan la producción de petróleo y gas por provincia (IAPG 2012).

Las actividades inherentes a la explotación hidrocarburífera ocasionan cambios adversos en el ambiente con riesgos para la salud y la propia industria La remediación de suelos contaminados con petróleo crudo, lodos de perforación y solventes derivados de la explotación es un tema de intensa investigación actualmente (Singh y Celin 2010, Alvaro et al. 2015). En este contexto, estudios realizados los últimos años indican que la presencia de hidrocarburos del petróleo modifica las propiedades físicas y químicas de los suelos (Martínez y López 2001) causando procesos de fototoxicidad (Meudec et al. 2006) y alteración en las comunidades microbianas (Maila et al. 2006), entre otros.

Debido a las características de los suelos de la región patagónica, las precipitaciones escasas y las bajas temperaturas, sólo algunos de los procesos de degradación o transferencia entre suelo, aire y agua cobran mayor relevancia (Ríos y Nudelman 2005). La volatilización es un proceso de transferencia de hidrocarburos que tiene importancia regional debido a los períodos de fuertes vientos. La movilidad de los componentes del petróleo depende de la permeabilidad del suelo y de la presencia de hidrocarburos livianos, generalmente más solubles y de baja viscosidad.

Las técnicas más recientes de evaluación de sitios contaminados con hidrocarburos se fundamentan en la metodología del análisis de riesgo y se basan en la valoración del efecto que producen agentes químicos presentes en un sitio contaminado en la salud humana y el ambiente debido a la sumatoria de riesgos individuales (ASTM 2010). La biorremediación es una tecnología emergente que utiliza las actividades metabólicas de microorganismos, hongos, plantas o las enzimas derivadas de ellos para degradar, transformar o remover contaminantes. La biorremediación de hidrocarburos del petróleo depende de diversos factores: presencia de una población microbiana activa, estructura y concentración del contaminante, disponibilidad de oxígeno y nutrientes, tipo de suelo, cantidad de materia orgánica (MO) y minerales presentes en el mismo, entre otros. En este sentido, las condiciones ambientales y las características físicas 
y químicas del suelo afectan la población microbiana nativa existente, por lo cual su estudio constituye un requisito previo para adecuar el crecimiento bacteriano a condiciones de laboratorio.

Estudios recientes realizados en pasivos ambientales con altas concentraciones de hidrocarburos (Alvaro et al. 2014) y en suelos de diferentes características edafológicas de la Norpatagonia Argentina contaminados con petróleo (Alvaro et al. 2015) han demostrado la eficacia de la bioestimulación utilizando enmiendas orgánicas e inorgánicas. La biorremediación de suelos contamindos con hidrocarburos de petróleo mediante la aplicación de enmiendas orgánicas es una práctica de escasa aplicación en esta región a nivel experimental.

Durante el tratamiento de aguas residuales se genera material sólido sedimentable llamado lodos cloacales o biosólidos (B). Los B están constituidos por materia orgánica fermentable, minerales inertes, nutrientes y poseen alto contenido de humedad (Norma 2002). Debido a su elevado porcentaje de materia orgánica (MO) y nutrientes, pueden ser utilizados como enmiendas después de someterlos a un proceso de estabilización. Su utilización está especialmente indicada en suelos arenosos y semidesérticos, tales como los de la meseta patagónica.

Considerando lo expuesto, en el presente trabajo se estudió, a escala de laboratorio, la tasa de degradación de los hidrocarburos totales del petróleo (HTP) y de distintas fracciones del mismo mediante la incorporación de $\mathrm{B}$, frente a la biorremediación convencional (aireación, laboreo y humectación) en un suelo regional típico contaminado con un $5 \%$ (p/p) de petróleo.

\section{MATERIALES Y MÉTODOS}

\section{Muestreo del suelo y enmienda utilizada}

Para la experiencia se recolectó un suelo de características geomorfológicas típicas de la meseta patagónica afectada por la actividad petrolera, perteneciente a la formación geológica Vaca Muerta de la Cuenca Neuquina. En esta región predominan los suelos de textura franco arenosa, arenosos y arcillosos, con alto contenido de carbonato de calcio, muy escasa $\mathrm{MO}, \mathrm{pH}$ moderadamente alcalino y un alto déficit hídrico anual (Alvaro et al. 2015). El sitio de extracción fue escogido considerando que no se encuentran locaciones de explotación hidrocarburífera en el área circundante, si bien dicho suelo posee las mismas características morfológicas de los suelos de exploración y explotación.
Las muestras de suelo se tomaron entre los 0 y $30 \mathrm{~cm}$ de profundidad, despreciando la cubierta vegetal y se secaron a temperatura ambiente, sobre bandejas metálicas, durante $48 \mathrm{~h}$. Se eliminaron elementos indeseables, se homogeneizaron con mortero y se tamizaron mediante malla metálica de $2 \mathrm{~mm}$.

En la región los B se utilizan con fines agrícolas. Los B utilizados se obtuvieron de las eras de secado de la planta de tratamiento de efluentes cloacales de la ciudad de Neuquén, operada por el Ente Provincial de Agua y Saneamiento de la Provincia de Neuquén (EPAS).

\section{Análisis físicos, químicos y bacteriológicos}

Los análisis físicos y químicos de las muestras de suelo se realizaron por triplicado utilizando métodos estándar (Sparks 2003). Las determinaciones y técnicas utilizadas fueron: carbono orgánico y materia orgánica (método de Walkley-Black), textura (método de Bouyoucus), $\mathrm{pH}$ (método potenciométrico en pasta saturada $(\mathrm{pHp}))$, conductividad eléctrica (puente de Whiston en extracto de saturación del suelo), capacidad de intercambio catiónico y cationes totales (método de saturación por acetato de amonio), fósforo (método Olsen), retención hídrica $(\mathrm{RH})$ a capacidad de campo (método membrana de Richard).

En B, las determinaciones y métodos utilizados fueron: nitrógeno total (método Kjeldahl), fósforo total (método estándar 4500-P-F), hidrocarburos totales (método TPHCWG 1998b), aceites y grasas (método EPA 413.1), arsénico, cadmio, zinc, cobre, cromo total y mercurio (método EPA 3051A), níquel y plomo (método EPA $3050 \mathrm{~B} / 7000 \mathrm{~B}$ ), bifenilos policlorados (método EPA 3051A), Escherichia coli (recuento del número de bacterias viables por dilución y siembra en placa), microorganismos heterótrofos aerobios totales y degradadores de hidrocarburo (método Número Más Probable (NMP)) (Woomer 1994).

Para el recuento de los microorganismos heterótrofos aerobios totales (HAT) se utilizó un medio de cultivo completo, que provee una fuente de carbono de rápida asimilación junto con factores de crecimiento. En cambio, para la determinación de los microorganismos degradadores de hidrocarburos (DH), se utilizó un medio de cultivo mínimo, consistente en una solución salina, con el agregado de micronutrientes y queroseno como única fuente de carbono y energía. La cuantificación de benceno, tolueno y xilenos (BTEX), se realizó mediante el método EPA 5021 A "Compuestos orgánicos volátiles en suelos y otras matrices sólidas, según el análisis de equilibrio de espacio en cabeza" acoplada a 
cromatografía gaseosa. La determinación de las distintas familias de hidrocarburos presentes en el suelo contaminado se realizó mediante el método ASTM D-5739/00, "Práctica estándar para la identificación de derrames de petróleo por cromatografía gaseosa y espectrometría de masas de baja resolución por impacto electrónico", que se utiliza para realizar una comparación cualitativa (generalmente llamada de huella digital) de los componentes del petróleo en las distintas etapas del proceso de degradación. La cuantificación de los materiales extraíbles con $n$-hexano (HEM) y los hidrocarburos de fracción pesada (HFP) se realizó siguiendo la Norma NMX-AA-134-SCFI (2006). La concentración de hidrocarburos totales del petróleo en suelo (HTP) se determinó por el método TPHCWG (1998b.)

\section{Diseño experimental de biorremediación}

El ensayo de laboratorio se realizó en microcosmos durante un periodo de 120 días e involucró dos tratamientos de biorremediación con tres repeticiones cada uno y un diseño completamente aleatorizado. Los microcosmos se armaron en recipientes de vidrio, con seis $\mathrm{kg}$ de suelo cada uno. Una semana antes de iniciar cada tratamiento se contaminó el suelo con petróleo crudo al $5 \%(\mathrm{p} / \mathrm{p})$. La homogeneización del suelo con el hidrocarburo se hizo en recipientes plásticos, mediante agitación y mezcla lenta de todo el material a fin de minimizar la volatilización de hidrocarburos, dos veces por semana. Los tratamientos ensayados fueron:

Tratamiento SC: el suelo contaminado con $5 \%$ de petróleo $(\mathrm{p} / \mathrm{p})$ sólo recibió laboreo, aireación y aporte de agua. Tratamiento SB: el suelo contaminado con $5 \%$ de petróleo $(\mathrm{p} / \mathrm{p})+\mathrm{B}$. La dosis de B agregada fue de $40 \mathrm{~g} / \mathrm{kg}$ de suelo (base seca).

Durante el periodo en el que se desarrolló el trabajo se mantuvieron las siguientes condiciones ambientales para los tratamientos: el aporte de agua en cada microcosmos se realizó dos veces por semana, incorporando el volumen necesario para alcanzar el $80 \%$ de la capacidad de campo del suelo. La temperatura ambiente en el laboratorio osciló en el rango $20 \pm 4{ }^{\circ} \mathrm{C}$.

\footnotetext{
Análisis estadístico

Se utilizó el paquete estadístico InfoStat, versión 2011e (Di Renzo et al. 2011) para realizar un análisis de regresión no lineal para las tres repeticiones de cada tratamiento y un análisis de la varianza para comprobar si había diferencias entre las velocidades medias de degradación de hidrocarburos entre tratamientos.
}

\section{RESULTADOS}

\section{Análisis físicos y químicos del suelo testigo}

De acuerdo con los análisis físicos y químicos obtenidos el suelo se clasificó como moderadamente alcalino ( $\mathrm{pH} 8.45 \pm 0.2$ ), con bajo contenido de carbono orgánico $(\mathrm{CO}=0.10 \pm 0.1 \%), \mathrm{MO}$, $(0.15 \pm 0.08 \%)$, nitrógeno $(0.01 \pm 0.005 \%)$ y medianamente provisto en fósforo $(13.8 \pm 0.2 \mathrm{ppm})$, baja capacidad de intercambio de catiónico $(\mathrm{CIC}=$ $11.6 \pm 0.2 \mathrm{mEq} / 100 \mathrm{~g}$ ), retención hídrica a $0.3 \mathrm{~atm}$ $\mathrm{RH}=3.30 \pm 0.2 \%$, retención hídrica a $15 \mathrm{~atm} \mathrm{RH}$ $=1.80 \pm 0.4 \%$. Considerando los resultados de conductividad eléctrica $(\mathrm{CE})=0.60 \pm 0.2 \mathrm{dS} / \mathrm{m}$ y el porcentaje de sodio intercambiable (PSI) $=5.80 \pm$ $0.1 \%$ se catalogó como suelo normal, dentro de los rangos frecuentes para la región (Ríos y Nudelman 2005). Este tipo de suelos posee características beneficiosas y a la vez perjudiciales para los procesos de biorremediación. Por un lado, la clase textural franco arenosa favorece la circulación de agua y aire. En contraste, posee una baja retención de agua, que sumado a la hidrofobicidad de los hidrocarburos adsorbidos a las partículas del suelo demandan un aumento en la frecuencia de humectación (Adams et al. 2011). Otra desventaja es el bajo contenido de MO y nutrientes contenidos en este tipo de suelos, que determinan una actividad microbiana muy disminuida (Adams Schroeder et al. 2002).

\section{Análisis químico y microbiológico del biosólido}

En el cuadro I se presentan los resultados obtenidos de la caracterización de los biosólidos. En la última columna se incorporaron los límites establecidos en la Resolución 97/2001 del Ministerio de Desarro1lo Social y Medio Ambiente, República Argentina, que aprueba el Reglamento para el Manejo Sustentable de Barros Generados en Plantas de Tratamiento de Efluentes Líquidos en el ámbito federal. Estos resultados establecieron que los biosólidos utilizados en el estudio son aptos para uso agrícola, ganadero, forestal o para recuperación de suelos degradados, sin restricciones de tipo sanitario, debido a que el análisis de la composición química del biosólido para compuestos orgánicos o inorgánicos tipificados como tóxicos, así como el recuento de microorganismos se encuentran dentro de los límites A.1 permitidos, especificados en la Resolución 97/2001.

Resultados similares se informaron en ensayos realizados en varios países del mundo, por lo que su aporte controlado al suelo no traería aparejado un riesgo significativo al ambiente ni a los seres vivos (Maila y Cloete 2005). 
CUADRO I. ANÁLISIS QUÍMICO Y MICROBIOLÓGICO DEL BIOSÓLIDO

\begin{tabular}{lccc}
\hline Parámetros & Unidad & Valor & Límite A.1 \\
\hline Arsénico & $\mathrm{mg} / \mathrm{kg}$ & $<10.0$ & 75 \\
Cadmio & $\mathrm{mg} / \mathrm{kg}$ & $<1.0$ & $20-40$ \\
Zinc & $\mathrm{mg} / \mathrm{kg}$ & $144 \pm 0.3$ & $2500 \mathrm{a} 4000$ \\
Cobre & $\mathrm{mg} / \mathrm{kg}$ & $37.4 \pm 0.2$ & $1000 \mathrm{a} 1750$ \\
Cromo total & $\mathrm{mg} / \mathrm{kg}$ & $10.2 \pm 0.1$ & $1000 \mathrm{a} 1500$ \\
Mercurio & $\mathrm{mg} / \mathrm{kg}$ & $<0.8$ & $16 \mathrm{a} 25$ \\
Níquel & $\mathrm{mg} / \mathrm{kg}$ & $<5.0$ & $300 \mathrm{a} 400$ \\
Plomo & $\mathrm{mg} / \mathrm{kg}$ & $27.7 \pm 0.2$ & $750 \mathrm{a} 1200$ \\
Bifenilos policlorados & $\mathrm{mg} / \mathrm{kg}$ & $<0.5$ & 0.8 \\
Escherichia coli & $\mathrm{UFC} / \mathrm{g}$ & $<100$ & $<1000$ \\
Fósforo total & $\mathrm{mg} / \mathrm{kg}$ & $36.0 \pm 0.2$ & $\mathrm{~N} / \mathrm{E}$ \\
Nitrógeno total & $\mathrm{mg} / \mathrm{kg}$ & $4125 \pm 1.2$ & $\mathrm{~N} / \mathrm{E}$ \\
$\begin{array}{l}\text { Hidrocarburos } \\
\text { Grasas y aceites }\end{array}$ & $\mathrm{mg} / \mathrm{kg}$ & $941.1 \pm 0.6$ & $\mathrm{~N} / \mathrm{E}$ \\
$\begin{array}{l}\text { Microorganismos heterótrofos } \\
\text { aeróbicos totales }\end{array}$ & $\mathrm{mg} / \mathrm{kg}$ & $9934.3 \pm 2.5$ & $\mathrm{~N} / \mathrm{E}$ \\
$\begin{array}{l}\text { Microorganismos } \\
\text { degradadores de hidrocarburo }\end{array}$ & $\mathrm{N}^{\mathrm{o}} \mathrm{microorg} / \mathrm{g}$ suelo & & $\mathrm{N} / \mathrm{E}$ \\
\hline
\end{tabular}

Nota: N/E: no establecido. UFC: Unidades formadoras de colonia. Límites A.1: establecidos en la Resolución (2001) que aprueba el "Manejo sustentable de barros generados en plantas de tratamiento de efluentes líquidos" en la República Argentina

\section{Caracterización del petróleo}

De acuerdo con el peso molecular de los hidrocarburos presentes en el petróleo utilizado en la experiencia, los hidrocarburos de hasta 14 átomos de carbono correspondieron al $94.1 \% \mathrm{y}$ los de quince átomos de carbono y superiores al $5.9 \%$. La determinación de la gravedad específica del petróleo fue de 38 grados API, densidad $0.84 \mathrm{~g} / \mathrm{mL}$ y se clasificó como petróleo ligero. El porcentaje de las distintas familias de hidrocarburos del petróleo estudiado se muestra en el cuadro II y los componentes presentes según el número de átomos de carbono en el cuadro III, donde puede observarse que el mayor porcentaje de hidrocarburos está comprendido entre los que poseen de cinco a nueve átomos de carbono, correspondiendo con la clasificación anteriormente citada.

\section{Evolución del proceso de biorremediación}

Los resultados del tratamiento con humectación, aireación y laboreo indicados como tratamiento SC, así como las reducciones observadas en las fracciones HEM y HFP se observan en los cuadros IV y $\mathbf{V}$.

Los porcentajes de reducción de HEM y HFP para cada tratamiento se determinaron a intervalos regulares de tiempo, hasta los 120 días. Es importante recalcar que los HEM están constituidos por hidrocarburos no volátiles, aceites vegetales, grasas animales, ceras, jabones y materia relacionada (Norma NMX-AA-134-SCFI-2006), por lo que el parámetro apropiado para realizar el seguimiento del proceso degradativo de hidrocarburos son los HFP.

Al finalizar la experiencia los HEM mostraron una marcada reducción con el aporte de biosólidos respecto al tratamiento donde sólo se realizó aireación, laboreo y humectación (SC).

La misma tendencia se observa en la degradación de los HFP al finalizar la experiencia, siendo la reducción de hidrocarburos estadísticamente significativa. En el tratamiento con aporte de materia orgánica, la degradación de HEM y HFP se produjo principalmente durante los primeros ochenta días de iniciada la biorremediación.

CUADRO II. FRACCIONES DEL PETRÓLEO DETERMINADAS POR EL MÉTODO ASTM D-5739/00 \pm DESVIACIÓN ESTÁNDAR

\begin{tabular}{lc}
\hline Componente & $\%$ /peso \\
\hline Total parafínicos & $32.0 \pm 0.2$ \\
Total nafténicos y de mayor PM & $28.2 \pm 0.2$ \\
Total mono-aromáticos & $5.40 \pm 0.2$ \\
Total isoparafínicos & $34.4 \pm 0.2$ \\
Total & 100 \\
\hline
\end{tabular}


CUADRO III. CARACTERIZACIÓN DE LOS COMPONENTES DEL PETRÓLEO SEGÚN EL NÚMERO DE ÁTOMOS DE CARBONO \pm DESVIACIÓN ESTÁNDAR.

\begin{tabular}{lc}
\hline Componente & \%/peso \\
\hline $\mathrm{C} 3$ & $0.23 \pm 0.02$ \\
$i \mathrm{C} 4$ & $1.10 \pm 0.02$ \\
$n \mathrm{C} 4$ & $2.97 \pm 0.02$ \\
$i \mathrm{C} 5$ & $7.10 \pm 0.02$ \\
$n \mathrm{C}-5$ & $6.70 \pm 0.02$ \\
$\mathrm{C} 6$ & $12.2 \pm 0.02$ \\
$\mathrm{C} 7$ & $23.6 \pm 0.02$ \\
$\mathrm{C} 8$ & $26.7 \pm 0.02$ \\
$\mathrm{C} 9$ & $14.0 \pm 0.02$ \\
$\mathrm{C} 10$ & $1.09 \pm 0.02$ \\
$\mathrm{C} 11$ & $0.70 \pm 0.02$ \\
$\mathrm{C} 12$ & $0.27 \pm 0.02$ \\
$\mathrm{C} 13$ & $0.003 \pm 0.001$ \\
$\mathrm{C} 14$ & $0.22 \pm 0.02$ \\
$\mathrm{C} 15$ & $0.20 \pm 0.02$ \\
$\mathrm{C} 16$ & $0.25 \pm 0.02$ \\
$\mathrm{C} 17$ & $0.34 \pm 0.02$ \\
$\mathrm{C} 18$ & $0.24 \pm 0.02$ \\
$\mathrm{C} 19$ & $0.30 \pm 0.02$ \\
$\mathrm{C} 20$ y superiores & $1.87 \pm 0.02$ \\
\hline
\end{tabular}

CUADRO IV. PORCENTAJE DE MATERIAL EXTRAÍBLE CON $n$-HEXANO DURANTE LA EXPERIENCIA EN EL TRATAMIENTO TESTIGO (SC) Y CON APORTE DE BIOSÓLIDOS (SB) \pm DESVIACIÓN ESTÁNDAR. NORMA (2006)

\begin{tabular}{ccc}
\hline Tiempo de muestreo (días) & SC (\% peso) & SB (\% peso) \\
\hline 0 & $1.67 \pm 0.2$ & $1.87 \pm 0.3$ \\
15 & $1.52 \pm 0.2$ & $1.47 \pm 0.2$ \\
30 & $1.40 \pm 0.3$ & $1.29 \pm 0.3$ \\
45 & $1.30 \pm 0.4$ & $1.13 \pm 0.3$ \\
80 & $1.19 \pm 0.4$ & $0.87 \pm 0.5$ \\
120 & $1.07 \pm 0.3$ & $0.65 \pm 0.4$ \\
$\%$ de reducción & $35.9 \pm 0.3$ & $65.2 \pm 0.33$ \\
\hline
\end{tabular}

CUADRO V. PORCENTAJE DE HIDROCARBUROS DE FRACCIÓN PESADA DURANTE LA EXPERIENCIA EN EL TRATAMIENTO TESTIGO (SC) Y CON APORTE DE BIOSÓLIDOS (SB) \pm DESVIACIÓN ESTÁNDAR. NORMA (2006)

\begin{tabular}{ccc}
\hline Tiempo de muestreo (días) & SC $(\%$ peso) & SB (\% peso $)$ \\
\hline 0 & $1.16 \pm 0.3$ & $1.61 \pm 0.4$ \\
15 & $1.00 \pm 0.3$ & $0.96 \pm 0.3$ \\
30 & $0.85 \pm 0.2$ & $0.77 \pm 0.2$ \\
45 & $0.72 \pm 0.2$ & $0.60 \pm 0.2$ \\
80 & $0.59 \pm 0.2$ & $0.36 \pm 0.3$ \\
120 & $0.48 \pm 0.2$ & $0.19 \pm 0.2$ \\
$\%$ de reducción & $58.6 \pm 0.23$ & $89.2 \pm 0.26$ \\
\hline
\end{tabular}

Determinación de sustancias extraíbles con n-hexano (HEM) e hidrocarburos de fracción pesada (HFP)

En los cuadros IV y $\mathbf{V}$ se presentan los valores obtenidos para ambos parámetros, en ellos se indica el promedio de los análisis realizados por triplicado para cada tratamiento, expresados en porcentaje.

En el cuadro $\mathbf{V}$ se observa que los resultados iniciales de HFP son considerablemente menores a los esperados al contaminar el suelo con un $5 \%$ de petróleo (p/p). Ello se debió probablemente a la composición del petróleo utilizado en la experiencia, datos con los cuales no se contaba al iniciar el trabajo. En el tratamiento SB se observó un porcentaje de reducción de HFP del $89.2 \%$ al finalizar el tratamiento, lo que indica una acentuada disminución de HFP en el tratamiento con biosólidos. Considerando la viabilidad de los biosólidos en el proceso de degradación, se realizó la determinación de las fracciones de hidrocarburos utilizando técnicas analíticas de mayor sensibilidad.

\section{Cuantificación de benceno, tolueno y xilenos (BTEX) por cromatografía gaseosa}

En el cuadro VI se puede apreciar que a los 14 días de iniciado el tratamiento se verificó una importante disminución en las concentraciones de BTEX, alcanzando el benceno y el tolueno los valores

CUADRO VI. CUANTIFICACIÓN DE BTEX EN LAS MUESTRAS DE SUELO HASTA LOS 14 DÍAS DE TRATAMIENTO \pm DESVIACIÓN ESTÁNDAR. MÉTODO EPA 5021 A

\begin{tabular}{|c|c|c|}
\hline \multicolumn{3}{|c|}{ Benceno (mcg/g peso seco) } \\
\hline Tiempo (días) & $\begin{array}{l}\text { Tratamiento } \\
\text { testigo }\end{array}$ & $\begin{array}{l}\text { Tratamiento } \\
\text { con biosólidos }\end{array}$ \\
\hline 0 & $7.08 \pm 0.3$ & $6.63 \pm 0.3$ \\
\hline 14 & $<0.05$ & $<0.05$ \\
\hline \multicolumn{3}{|c|}{ Tolueno (mcg/g peso seco) } \\
\hline Tiempo (días) & $\begin{array}{l}\text { Tratamiento } \\
\text { testigo }\end{array}$ & $\begin{array}{l}\text { Tratamiento } \\
\text { con biosólidos }\end{array}$ \\
\hline 0 & $84.4 \pm 0.3$ & $79.8 \pm 0.3$ \\
\hline 14 & 0.08 & $<0.05$ \\
\hline \multicolumn{3}{|c|}{ Xilenos totales (mcg/g peso seco) } \\
\hline Tiempo (días) & $\begin{array}{l}\text { Tratamiento } \\
\text { testigo }\end{array}$ & $\begin{array}{l}\text { Tratamiento } \\
\text { con biosólidos }\end{array}$ \\
\hline 0 & $82.7 \pm 0.4$ & $73.9 \pm 0.4$ \\
\hline 14 & $8.4 \pm 0.3$ & $3.8 \pm 0.3$ \\
\hline
\end{tabular}


permitidos para uso agrícola en ambos tratamientos. En el caso de los xilenos, los valores obtenidos en el tratamiento SB indicaron que los suelos son aptos para uso residencial, mientras que los obtenidos en el tratamiento SC los clasificaron como aptos para uso industrial, de acuerdo a lo establecido en la Ley
24051 de Residuos Peligrosos (República Argentina). Estos resultados concuerdan con los informados por diversos autores, quienes coinciden en que gran parte de los hidrocarburos monoaromáticos se volatilizan durante los procesos de aireación del suelo (Eweis et al. 1999, Margesin et al. 2003).

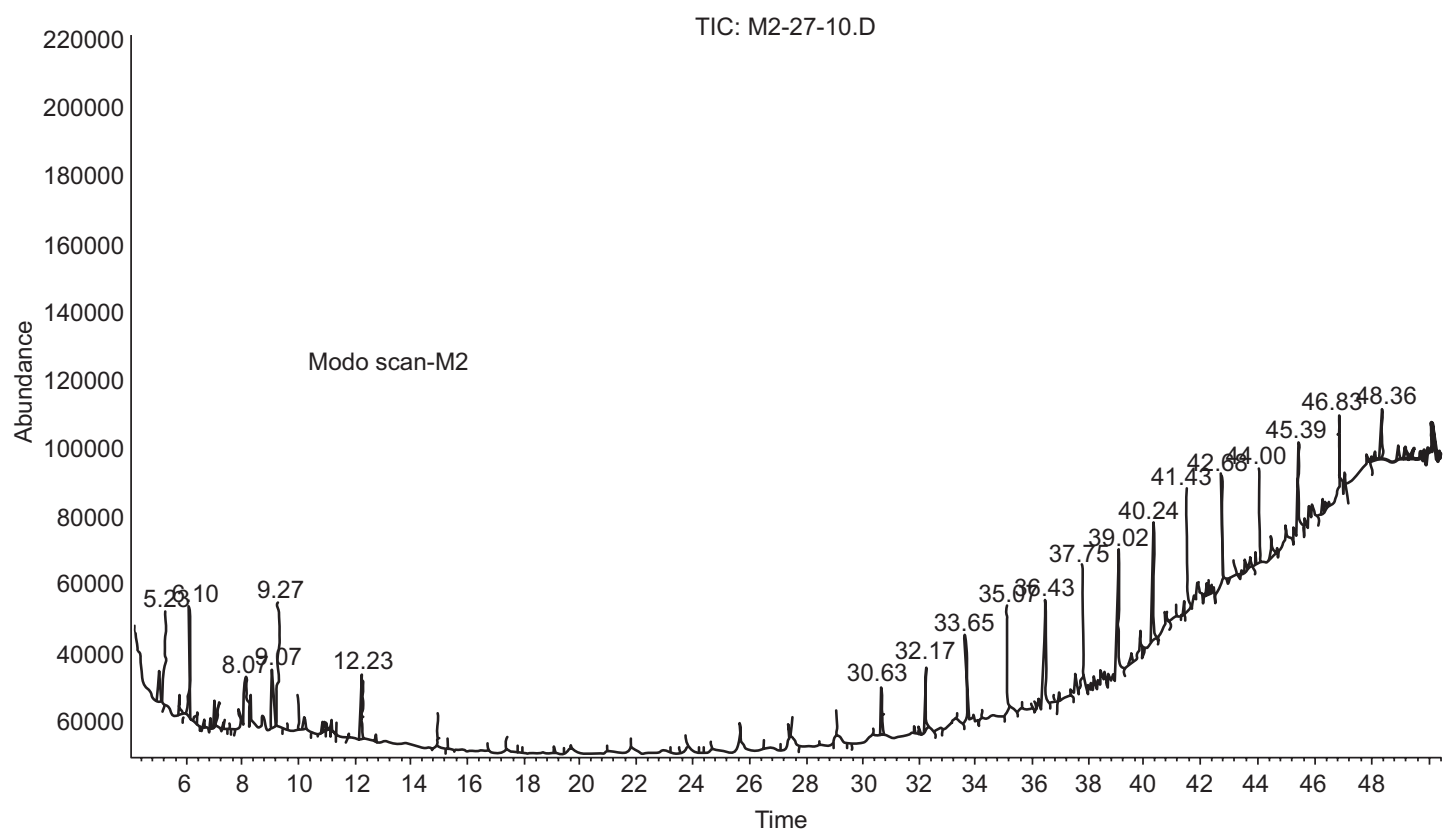

Fig. 1. Suelo contaminado previo al agregado de biosólidos. Cromatograma de iones totales (TIC, por sus siglas en inglés), en modo de escaneo. Tiempo 0 días

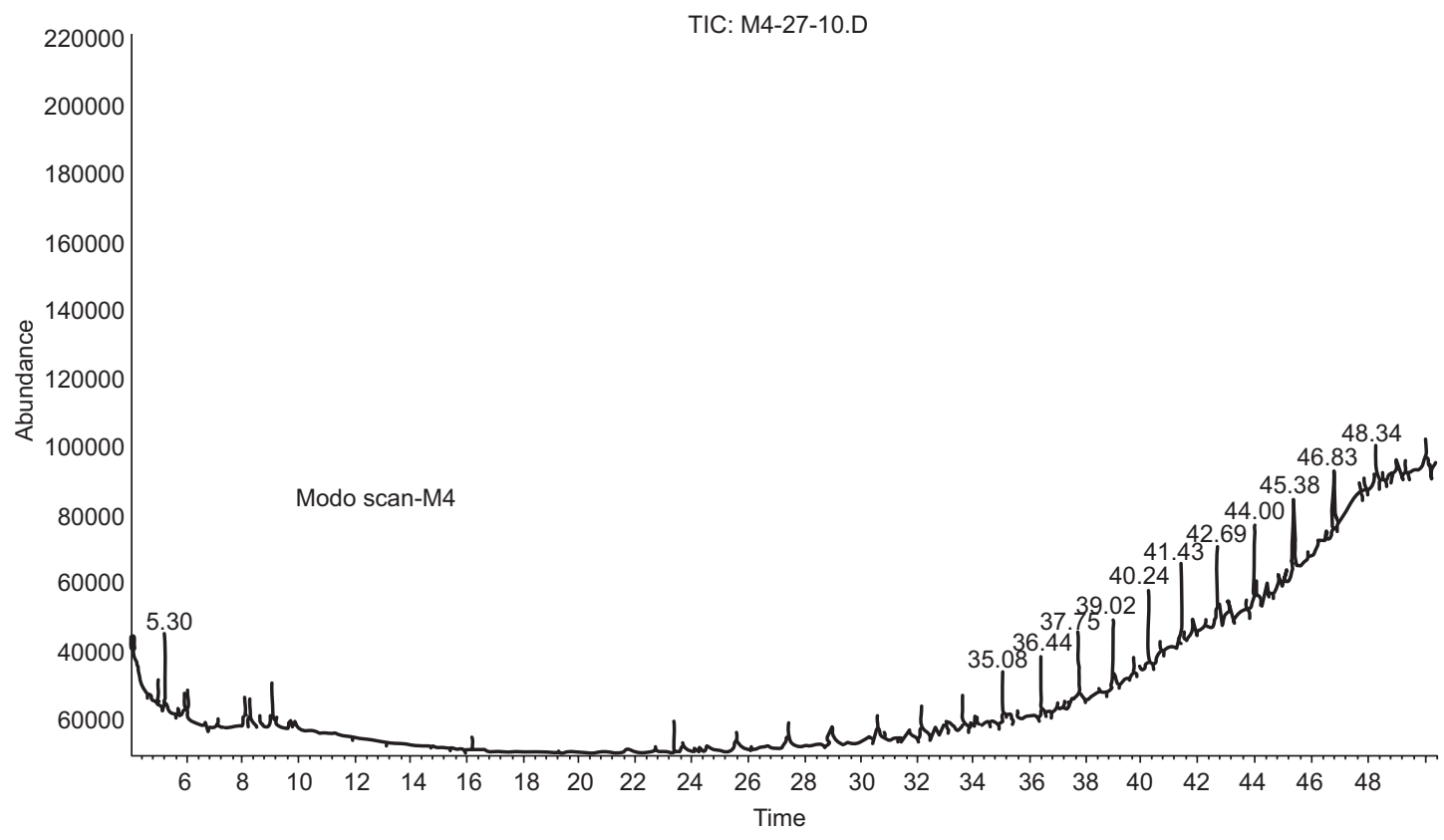

Fig. 2. Suelo contaminado previo al agregado de biosólidos. Cromatograma de iones totales (TIC, por sus siglas en inglés), en modo de escaneo. Tiempo 120 días 


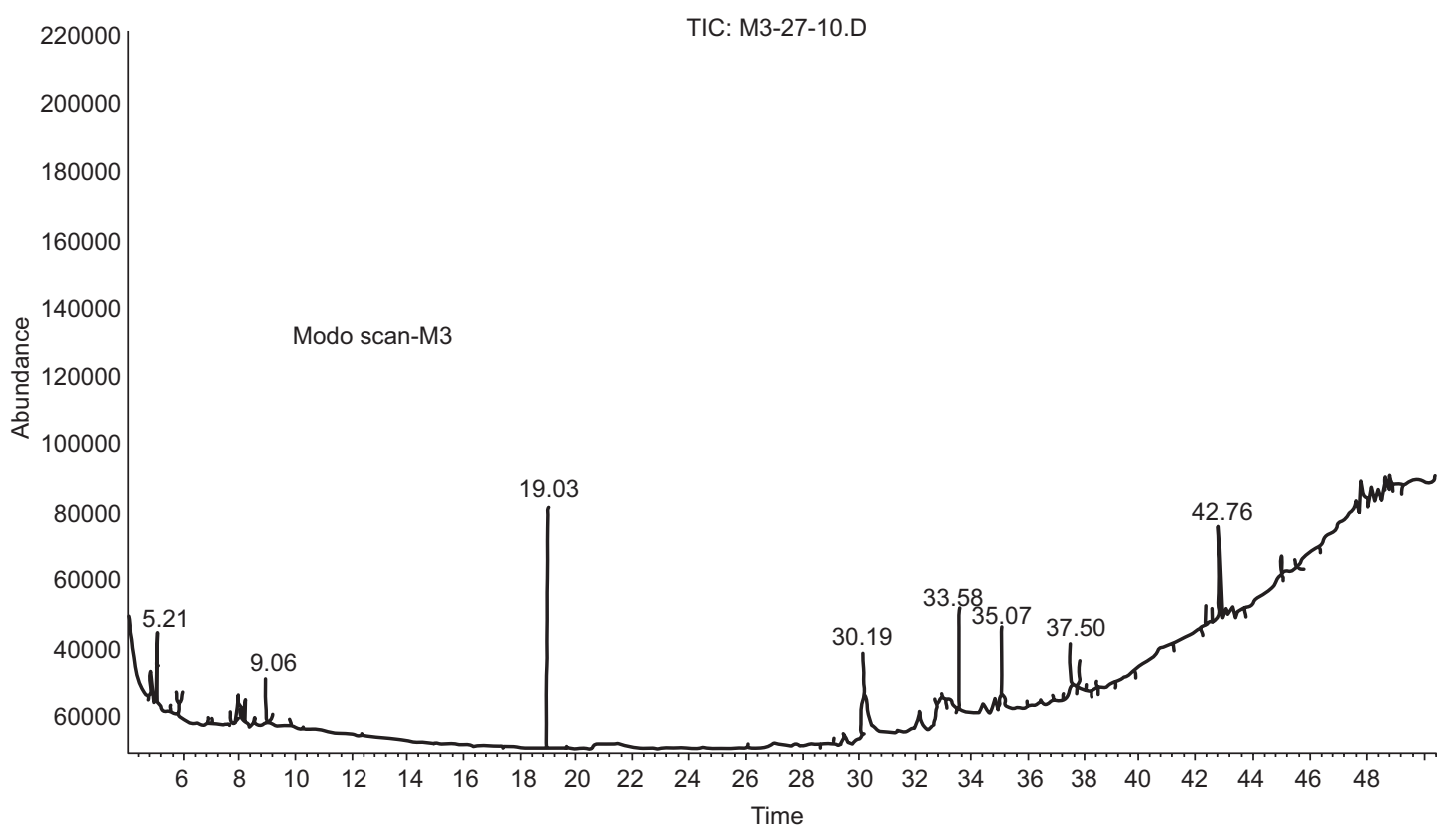

Fig. 3. Suelo contaminado con agregado de biosólidos. Cromatograma de iones totales (TIC, por sus siglas en inglés), en modo de escaneo. Tiempo 120 días

La fracción de hidrocarburos alifáticos y aromáticos de bajo PM fue degradada más rápidamente que las fracciones de hidrocarburos aromáticos policíclicos (HFP). Esto es debido a que la fracción alifática está formada principalmente por $n$-alcanos, hidrocarburos que son fácilmente metabolizados por los microorganismos (Ríos y Nudelman 2008). La utilización como fuente de carbono de compuestos orgánicos se dificulta cuando los contaminantes presentan ramificaciones y dos o más anillos aromáticos en su estructura.

Caracterización de las fracciones de hidrocarburos presentes en los suelos contaminados por cromatografía gaseosa y espectrometría de masas.

En la figura 1 se observa el cromatograma del suelo contaminado con petróleo previo al agregado de biosólidos. En las figuras $\mathbf{2}$ y $\mathbf{3}$ se observan los cromatogramas correspondientes a los 120 días para los tratamientos SC y SB, respectivamente. Superponiendo los cromatogramas correspondientes al inicio y al final del tratamiento se observa disminución de los hidrocarburos de bajo PM en ambos tratamientos y una marcada eliminación de picos correspondientes a hidrocarburos de elevado PM en el tratamiento SB. $\mathrm{Al}$ inicio del proceso hay una variedad de compuestos presentes en el suelo contaminado que corresponden a hidrocarburos de bajo PM como el benceno y el xileno (tiempo de retención $5.21 \mathrm{~s}$ ) y alto PM como el tetratetracontano de 44 átomos de carbono (tiempo de retención de $40.24 \mathrm{~s}$ ). A los 120 días se observa una clara disminución de los compuestos de bajo PM en ambos tratamientos. Esta tendencia se incrementa en SB, observándose en el cromatograma (Fig. 3) la eliminación de numerosos picos, variación en las señales de mayor tiempo de retención respecto al tratamiento testigo y la aparición de nuevos picos, probablemente debidos a compuestos presentes en los biosólidos y a sustancias ausentes al inicio del tratamiento, inherentes al metabolismo bacteriano. Si bien los antracenos y fenantrenos son resistentes a la degradación, se observa una sensible disminución en el número de picos de alto PM, lo cual indicaría que este tipo de compuestos han sido mayoritariamente degradados. Otro resultado a destacar comparando los cromatogramas del suelo contaminado sin agregado de enmienda (Fig. 1) y al finalizar los tratamientos (Figs. 2 y 3 ) es la disminución de picos de bajo PM correspondientes principalmente a alcanos en el tratamiento SC, tendencia que se acentúa en el tratamiento con agregado de $\mathrm{B}$.

\section{Análisis microbiológico}

El incremento de la población microbiana nativa del suelo puede acelerar la degradación de contaminantes orgánicos, para ello es necesario agregar nutrientes de manera equilibrada, fundamentalmente fuentes de carbono. Esta afirmación se basa en el 
CUADRO VII. NÚMERO MÁS PROBABLE (NMP) DE MICROORGANISMOS DEGRADADORES DE HIDROCARBUROS (DH) Y HETERÓTROFOS AEROBIOS TOTALES (HAT) EN EL SUELO TESTIGO (SC) Y CON AGREGADO DE BIOSÓLIDOS (SB) ADISTINTOS TIEMPOS DEL PROCESO DE BIORREMEDIACIÓN

\begin{tabular}{lcc}
\hline \multirow{2}{*}{ Muestra de suelo } & \multicolumn{2}{c}{ Microorganismos (NMP/g suelo) } \\
\cline { 2 - 3 } & HAT & DH \\
\hline SC (tiempo 0) & $1.2 \times 10^{3}$ & $1.5 \times 10^{1}$ \\
SC (60 días) & $3.0 \times 10^{6}$ & $7.0 \times 10^{5}$ \\
SC (120 días) & $5.0 \times 10^{4}$ & $5.0 \times 10^{4}$ \\
SB (60 días) & $1.0 \times 10^{7}$ & $1.2 \times 10^{6}$ \\
SB (120 días) & $4.0 \times 10^{6}$ & $3.0 \times 10^{6}$ \\
\hline
\end{tabular}

hecho de que el agregado de grandes cantidades de carbono (hidrocarburos) interrumpe el equilibrio natural de los nutrientes en el sistema causando una rápida disminución de otros (tal como $\mathrm{N}$ y $\mathrm{P}$ ), y por lo tanto reduce el crecimiento bacteriano (Martínez Prado et al. 2011).

El recuento de microorganismos heterótrofos aerobios totales (HAT) y degradadores de hidrocarburos $(\mathrm{DH})$ se obtuvo una concordancia del $95 \%$ entre los resultados de las tres repeticiones realizadas para cada recuento. Los valores presentados son el promedio de los valores obtenidos.

El cuadro VII muestra la fluctuación de las poblaciones de microorganismos HAT y DH a lo largo de la experiencia. Al comienzo de ambos tratamientos se observó baja cantidad de microorganismos HAT y DH. Estos valores son inferiores en dos o tres órdenes de magnitud a los recomendados para biorremediar suelos contaminados con hidrocarburos, aunque son normales para este tipo de suelos, coincidentes con trabajos anteriores realizados en suelos provenientes de la región patagónica (Alvaro et al. 2015). Este resultado es debido a que el suelo en estudio posee baja concentración de MO y nutrientes, escasa humedad durante gran parte del año y está sometido a una intensa radiación solar, todos factores desfavorables para el desarrollo microbiano.

A los 60 días en ambos tratamientos, se observó incremento de las poblaciones HAT y DH respecto al tiempo cero (Cuadro VII).

A los 120 días de tratamiento, el recuento de ambas poblaciones de microorganismos se mantuvo en el mismo orden de magnitud para el tratamiento SB. El tratamiento SC mostró un decaimiento de la población HAT y el tratamiento SB mantuvo constantes los valores para DH respecto a los obtenidos a los 60 días. Estos resultados podrían deberse a la formación de sustancias tóxicas producto del metabolismo bacteriano que modifican la tasa de crecimiento de los microorganismos.

Los resultados obtenidos pueden explicarse considerando que el tratamiento SB proporciona una rápida disponibilidad de nutrientes y carbono fácilmente degradable, lo cual se manifiesta en un acelerado crecimiento de ambas poblaciones de microorganismos. El recuento de los mismos muestra que el desarrollo microbiano es superior en el tratamiento $\mathrm{SB}$, indicando que el $\mathrm{N}$ y el $\mathrm{P}$ presentes en $\mathrm{B}$ son elementos críticos para el desarrollo microbiano en este tipo de suelos, resultado recientemente informado para suelos agrícolas mexicanos contaminados con hidrocarburos (Castro-Mancilla et al. 2013).

\section{DISCUSIÓN}

La degradación obtenida de HEM, HFP y principalmente los cromatogramas realizados en el tratamiento control (SC) y con agregado de enmienda orgánica (SB), muestran marcadas diferencias al inicio y al final del proceso de biorremediación, lo cual permite deducir que se han eliminado componentes pertenecientes a distintas fracciones del petróleo. Los hidrocarburos de alto peso molecular, de estructura química estable y resistentes a la biodegradación, mostraron reducciones significativas, siendo el tratamiento SB el más efectivo. La baja volatilidad y escasa solubilidad de los HFP en agua, así como la poca posibilidad de migración por lixiviación fuera del sistema, indicarían que la eliminación de los mismos se debió principalmente al metabolismo microbiano. Los hidrocarburos alifáticos y aromáticos de bajo PM fueron eliminados rápidamente en suelos areno-francos sometidos a tratamientos de biorremediación debido a procesos de volatilización y degradación biológica.

El recuento de microorganismos heterótrofos aerobios totales y degradadores de hidrocarburos al inicio de ambos tratamientos fue bajo, probablemente debido a las propiedades físicas y químicas del suelo antes mencionadas. Sin embargo, la aplicación de biosólidos al suelo contaminado produjo un rápido aumento de la población microbiana lo que favoreció el proceso de biorremediación.

Dada la aparición de nuevos compuestos durante el tratamiento con biosólidos (Fig. 3), consideramos necesario evaluar la ecotoxicidad de los mismos, lo que permitiría reforzar criterios de seguimiento y 
determinación del punto de final del proceso. En este sentido, se continuará el estudio utilizando diferentes tipos de gramíneas y/o arbustos de la zona norpatagónica como especies fitoindicadoras del proceso de remediación aplicado.

\section{CONCLUSIONES}

En el presente trabajo se determinó la viabilidad de biorremediar suelos patagónicos contaminados con hidrocarburos mediante el agregado de biosólidos provenientes de plantas de tratamiento de aguas residuales. La baja concentración de materia orgánica y nutrientes inorgánicos disponibles en el suelo para el crecimiento bacteriano mejoró con el aporte del agregado de la enmienda aplicada.

Es interesante notar que debido a las características de los suelos patagónicos, asociado con el clima desértico de la región, el tratamiento con biosólidos resultó eficaz frente a los procesos convencionales de biorremediación, tales como aporte de F y laboreo, aireación y humectación (SC). Los resultados obtenidos fomentan la aplicación de este tratamiento en ensayos de campo para evaluar la sensibilidad del modelo a las variaciones de los principales factores que intervienen. Las reducciones observadas en el contenido de HFP confirman estas suposiciones. Asimismo, al tomar en cuenta la urgencia de dar un destino ambientalmente sustentable a los biosólidos generados en la región, los resultados del presente trabajo pueden contribuir a la selección de enmiendas orgánicas empleadas para sanear suelos contaminados y aumentar la efectividad del proceso en función de las propiedades del remediante aportado.

\section{REFERENCIAS}

Adams Schroeder R.H., Domínguez Rodríguez V.I. y Vinalay Carrillo L. (2002). Evaluation of microbial respiration and ecotoxicity in contaminated soils representative of the petroleum producing region of southeastern Mexico. Terra 20 (3), 253-265.

Adams R.H., Kanga-Leyva K., Guzmán-Osorio F.J. y Escalante-Espinosa E. (2011). Comparison of moisture management methods for the bioremediation of hydrocarbon contaminated soil. Afr. J. Biotechnol. 10 (3), 394-404.
Alvaro C.E.S., Olave A.J., Schmid P.M. y Nudelman N.S. (2014). Microbial remediation performance of environmental liabilities from oil spills in Patagonian soils: A laboratory-scale. Int. J. Res. Chem. Environ. 4 (3), 119-126.

Alvaro C.E.S., Torre A.M. y Nudelman N.S. (2015). Hydrocarbons degradation in crude-oil polluted $\mathrm{Pa}-$ tagonian soils with different organic matter quality: Effect of added organic amendment. Int. J. Res. Chem. Environ. 5 (2), 18-25.

ASTM (2000). Standard practice for oil spill identification by gas chromatography and positive ion electron impact mass spectrometry (D-5739/00). American Society for Testing and Materials. West Conshohocken, EUA, 13 pp.

ASTM 2010b (2010). Standard guide for risk-based corrective action applied at petroleum release sites E1739-95. American Society for Testing and Materials. Manual. West Conshohocken, EUA, 53 pp.

Castro-Mancilla Y.V., Castro-Meza B.I., de la GarzaRequena F.R., Rivera Ortiz P., Heyer-Rodríguez L. y Ortiz-Carrizales Y.P. (2013). Variación de las poblaciones microbianas del suelo por la adición de hidrocarburos. Terra 31 (3), 221-230.

Di Renzo J.A., Casanoves F., Balzarini M.G., González L. y Tablada M. (2011). InfoStat versión 2011e liberada. Grupo InfoStat. Facultad de Ciencias Agrarias. Universidad Nacional de Córdoba, Argentina [en línea]. http:// www.infostat.com.ar/index.php? $\bmod =$ page $\& \mathrm{id}=28$ $15 / 09 / 2015$

Eweis J.B., Ergas S.J., Chang D.P.Y. y Schroeder E.D. (1999). Principios de Biorrecuperación: Tratamientos para la descontaminación y regeneración de suelos y aguas subterráneas mediante procesos biológicos y fisicoquímicos. Mc Graw Hill/ Interamericana de España. Madrid, España, 327 pp.

IAPG (2012). Producción de petróleo por provincia. Instituto Argentino del Petróleo y del Gas [en línea]. http:/www.iapg.org.ar/sectores/estadisticas/productos/ listados/mayo12.htm 13/09/2015

Ley Nacional (1992). Ley Nacional 24051. Régimen de residuos peligrosos. Boletín Oficial de la República Argentina. 17 de enero de 1992.

Lladó S., Solanas A.M., Lapuente J., Borras M. y Viñas M. (2012). A diversified approach to evaluate biostimulation and bioaugmentation strategies for heavy-oil contaminated soil, Sci. of the Total Environ. 435-436, 262-269.

DOI: 10.1016/j.scitotenv.2012.07.032

Maila M.P. y Cloete T.E. (2005). Bioremediation of petroleum hydrocarbons through landfarming: Are simplicity and cost-effectiveness the only advantages? Env. Science and Biotechnology 3 (4), 349-360. 
Maila M.P., Randima P., Dronen K. y Cloete T. E. (2006). Soil microbial communities: Influence of geographic location and hydrocarbon pollutants. Soil Biol. Biochem. 38 (2), 303-310.

DOI: $10.1016 /$ j.soilbio.2005.05.006

Margesin R., Walder G. y Schinner S.F. (2003). Bioremediation Assessment of a BTEX-contaminated soil. Acta Biotechnol. 23 (1), 29-36.

Martínez M.V.E. y López F.S. (2001). Efecto de hidrocarburos en las propiedades físicas y químicas de suelo arcilloso. Terra 19 (1), 9-17.

Martínez-Prado A., Pérez-López M.E., Pinto-Espinoza J., Gurrola-Nevárez B.A. y Osorio-Rodríguez A.L. (2011). Biorremediación de suelo contaminado con hidrocarburos empleando lodos residuales como fuente alterna de nutrientes. Rev. Int. Contam. Ambie. 27 (3), 241-252.

Meudec A., Dussauze J., Deslandes E. y Poupart N. (2006). Evidence for bioaccumulation of PAHs within internal shoot tissues by a halophytic plant artificially exposed to petroleum polluted-sediments. Chemosphere 65 (3), 474-481. DOI: 10.1016/j.chemosphere.2006.01.058

Norma (2002). Norma NMX-004-SEMARNAT. Protección ambiental. Lodos y biosólidos. Especificaciones y límites máximos permisibles de contaminantes para su aprovechamiento y disposición final. Diario Oficial de la Federación, Ciudad de México, México, 56 pp. 15 de agosto de 2003.

Norma (2006). Norma NMX-AA-134-SCFI. SuelosHidrocarburos de fracción pesada por extracción y gravimetría. Método de prueba. Norma de la Secretaría de Economía de México. Programa Nacional de Medio Ambiente y Recursos Naturales 2001-2006. Ciudad de México, México, 27 pp. 12 de octubre de 2006.

Resolución (2001). Resolución 97/01 del Ministerio de Desarrollo Social y Medio Ambiente de la Nación Argentina. Manejo sustentable de barros generados en plantas de tratamiento de efluentes líquidos. Boletín Oficial de la Nación Argentina. 28 de noviembre de 2001.
Ríos S.M. y Nudelman N.S. (2005). Multilayer sorption model for the interactions between crude oil and clay in Patagonian soils. J. Disper. Sci. Technol. 26 (1), 19-25. DOI: 10.1081/DIS-200040228

Ríos S.M. y Nudelman N.S. (2008). Natural attenuation of oil spills in Patagonian Soils. Characterization by ${ }^{1} \mathrm{H}$ NMR spectroscopy. Environ. Technol. 29 (1), 23-33. DOI: $10.1080 / 09593330802008446$

Sparks D.L. (2003). Environmental soil chemistry. 2a ed. Academic Press. Boston, EUA, 352 pp.

Singh R. y Celin S.M. (2010). Biodegradation of BTEX compounds by bacterial strain under aerobic conditions. J. of Ecobiotechnol. 2 (4), 27-32.

Trindade P.V.O., Sobral L.G., Rizzo A.C.L., Leite S.G.F. y Soriano A.U. (2005). Bioremediation of a weathered and a recently oil-contaminated soils from Brazil: a comparison study. Chemosphere 58 (4), 515-522.

TPHCWG (1998). Analysis of petroleum hydrocarbons in environmental media. Total Petroleum Hydrocarbon Criteria Working Group Series. Volume 1, Amherst Scientific Publisher. Massachussets, EUA, 98 pp.

USEPA (1986). Total petroleum hydrocarbons in analytical methods and laboratories petrochemicals-oils. United States Environmental Protection Agency. Manual. Washington D. C., EUA, 111 pp.

USEPA (2014). Método 5021 Rev. 2 SW-846 Update V Test methods Manual. Volatile organic compounds in soils and other solid matrices using equilibrium headspace analysis. United States Environmental Protection Agency, $31 \mathrm{pp}$.

Walkley A. y Black L.A. (1934). An examination of Degtjareff method for determining soil organic matter and a proposed modification of the chromic acid titration method. Soil Sci. 37 (1), 29-38 pp.

Woomer P.L. (1994). Most probable number counts. En: Methods of soils analysis Part 2. Microbiological and biochemical properties. (S. H. Mickelson y J. M. Bigham, Eds.) Soil Science Society of America Book Series. Madison, EUA, pp. 59-79. 